"This is the peer reviewed version of the following article: Adv. Synth. Catal. 2015, 357, 2849-2854 which has been published in

final form at

http://onlinelibrary.wiley.com/doi/10.1002/adsc.201500635/abstract]. This article may be used for non-commercial purposes

in accordance with Wiley Terms and Conditions for Self-Archiving ."

\title{
Aluminium Catalysed Oxazolidinone Synthesis and their Conversion into Functional Non-Symmetrical Ureas
}

Victor Laserna, ${ }^{a}$ Wusheng Guo ${ }^{\mathrm{a}}$ and Arjan W. Kleij ${ }^{\mathrm{a}, \mathrm{b}}$ *

a Institute of Chemical Research of Catalonia (ICIQ), Av. Països Catalans 16, 43007 - Tarragona, Spain

Fax; (+34)-977920823; Tel.: (+34)-977920247; E-mail: akleij@iciq.es

b Catalan Institute of Research and Advanced Studies (ICREA), Pg. Lluís Companys 23, $08010-$

Barcelona, Spain

Received: ((will be filled in by the editorial staff))

Supporting information for this article is available.

\begin{abstract}
An efficient and practical aluminium-
catalysed approach towards a range of functional

oxazolidinones is reported. The method is based on

cheap and readily available starting materials including

terminal and internal (bicyclic) epoxides and

phenylcarbamate. The oxazolidinones serve as highly

useful synthons for the high yield preparation of

nonsymmetrical ureas by nucleophilic ring-opening

affor-
\end{abstract}

ding the targeted urea compounds with excellent functional group diversity, and high regio-selectivity and isolated yields up to $>99 \%$.

Keywords: aluminium; N,O-ligands; homogeneous catalysis; oxazolidinones; ureas

\section{Introduction}

Organic ureas are ubiquitous structures in organic chemistry and their re-emerged application potential in various areas of chemistry has been recently highlighted. ${ }^{[1]}$ Prominent fields where ureas have gained importance include (asymmetric) organocatalysis ${ }^{[2,3]}$ and supramolecular chemistry. ${ }^{[4]}$ Organocatalytic activation of organic substrates by means of hydrogen-bonding patterns that comprise of the two urea $\mathrm{NH}$ groups has been recognized as a powerful tool to orientate carbonyl fragments in order to increase the efficiency of various organic conversions. Asymmetric ureas have also been developed successfully and applied as enantiocontrolling mediators in, for instance, the asymmetric Strecker reaction. ${ }^{[5]}$ In the context of supramolecular applications, $N, N^{\prime}$-disubstituted urea derivatives have proven to be versatile building blocks for the preparation of hydrogen-bonded supramolecular polymers, ${ }^{[6,7]}$ helical type foldamers ${ }^{[8]}$ and anion transporting molecules. ${ }^{[9]}$

There exist various synthetic methods towards nonsymmetrical ureas although in most cases reactive species such as carbonylimidazolide ${ }^{[10]}$ or (in situ prepared) isocyanate reagents ${ }^{[11-13]}$ are required together with air-sensitive/toxic additives ${ }^{[14,15]}$ and/or expensive metal catalysts/ligands. ${ }^{[12]}$ Therefore, we set out to explore a practical synthesis of nonsymmetrical ureas from readily available and cheap starting materials combined with a highly modular nature of the reaction partners (see Scheme 1). The approach uses oxazolidinones (cyclic carbamates) as intermediates that allow for nucleophilic ring-opening by suitable amines. Such an approach is not completely without precedence, ${ }^{[16,17]}$ however so far oxazolidinone type intermediates have been rarely used for the preparation of nonsymmetrical ureas with wide 
scope $\mathrm{e}^{[18-20]}$ and thus a more general methodology based on these precursors derived from readily available, cheap epoxides and phenylcarbamate (Scheme 1) would provide an attractive and simple route towards highly functional ureas.
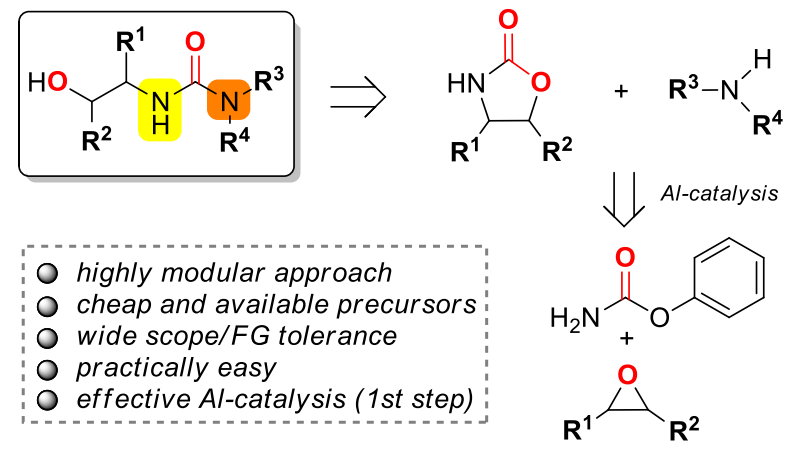

Scheme 1. A modular approach towards nonsymmetrical ureas through oxazolidinone intermediates.

Jacobsen et al. recently disclosed a method for the formation of chiral amino-alcohols through a Co(salen) mediated enantio-selective conversion of meso-cyclic epoxides in the presence of phenylcarbamate as nucleophile. ${ }^{[21]}$ This approach demonstrated the use of phenylcarbamate as a cheap and readily available reagent in the formal transfer of an "amide" unit to an epoxide giving enantio-enriched oxazolidinones as intermediates towards the synthesis of chiral amino-alcohols. However, the reported method was restricted to a limited number of cyclic epoxides. In order to be able to develop a more general method towards oxazolidinone precursors en route to nonsymmetrical ureas, we envisioned that our previously reported Lewis acidic $\mathrm{Al}($ aminotriphenolate) catalysts would present an alternative catalyst for oxazolidinone formation (Scheme 1). Recently we showed that both internal as well as terminal epoxides are easily activated towards nucleophilic ring-opening by these catalyst systems to form highly functional cyclic carbonates in the presence of carbon dioxide. ${ }^{[22-24]}$ In this work we will show that these Al-catalysts provide easy access to a wide range of oxazolidinone precursors useful for a modular approach towards highly functionalized ureas that are generally obtained in excellent yield and regio-selectivity.

\section{Results and Discussion}

We first set out to test a small series of $\mathrm{Al}$ (aminotriphenolate) complexes A-C (Figure 1) in the synthesis of oxazolidinone 1 under various conditions (Table 1 ) using the coupling of cyclohexene oxide ( $\mathrm{CHO}$ ) and phenylcarbamate as a benchmark reaction. The reaction without the addition of catalyst does not proceed at $50^{\circ} \mathrm{C}$ (entry 1 ), and at $70 \circ \mathrm{C}$ only low conversion of the phenylcarbamate reagent is noted. In general we found that those reactions that were carried out at this latter temperature (entries 2, 5-8 and 14-15) caused (partial) decomposition of the carbamate reagent and the white precipitates noted could be isolated and assigned to the formation of 1,3,5triazine-2,4,6(1H, 3H, 5H)-trione, a cyclic structure supported by ${ }^{1} \mathrm{H},{ }^{15} \mathrm{~N},{ }^{13} \mathrm{C}$ NMR and IR analysis (see Supporting Information).

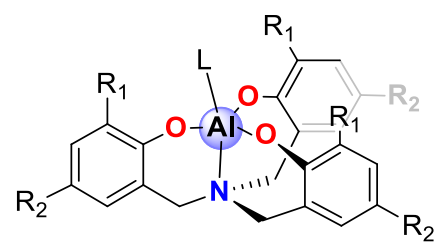
$A: R^{1}=R^{2}=M e ; L=$ none
B: $R^{1}=R^{2}=t B u ; L=$ thf
C: $R^{1}=R^{2}=C l ; L=$ thf

Figure 1. Al-catalysts A-C used in this work.

Therefore, we decided to optimize further the catalysis conditions at $50 \circ \mathrm{C}$ using various catalysts, catalyst loadings and amount of the epoxide (CHO). Al-catalyst A performs much better than B (cf., entries 5-8) but its use results in moderate substrate conversion levels at $50^{\circ} \mathrm{C}$ (entries 3 and 4). Much better activity was noted for the chloride-substituted Al-complex $\mathbf{C}$ for which a loading of $2.5 \mathrm{~mol} \%$ and an equimolar amount of epoxide (entry 11) already provided $81 \%$ conversion. Further increasing the epoxide/carbamate ratio to 2 led to nearly full conversion (95\%) with a high isolated yield (89\%) for the oxazolidinone product (entry 9). Therefore, the reaction conditions of this latter entry seem to be rather ideal for the synthesis of oxazolidinone 1 . 


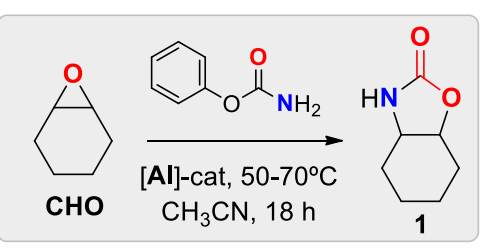

Table 1. Screening of catalyst structures $\mathbf{A}-\mathbf{C}$ and reaction conditions in the synthesis of oxazolidinone 1 from cyclohexene oxide and phenylcarbamate. ${ }^{\text {[a] }}$

\begin{tabular}{|c|c|c|c|c|c|}
\hline Entry & Cat. & $\begin{array}{l}\text { Amount } \\
\text { [mol\%] }\end{array}$ & $\begin{array}{l}\mathrm{CHO} \\
{[\mathrm{mmol}]}\end{array}$ & $\begin{array}{l}\mathrm{T} \\
{\left[{ }^{\circ} \mathrm{C}\right]}\end{array}$ & $\begin{array}{l}\text { Conv. }^{[b]} \\
{[\%]}\end{array}$ \\
\hline 1 & - & - & 1.0 & 50 & 0 \\
\hline 2 & - & - & 1.0 & 70 & $17^{[\mathrm{c}]}$ \\
\hline 3 & A & 2.5 & 1.0 & 50 & 41 \\
\hline 4 & A & 5.0 & 1.0 & 50 & 47 \\
\hline 5 & A & 2.5 & 1.0 & 70 & $94^{[\mathrm{c}]}$ \\
\hline 6 & A & 5.0 & 1.0 & 70 & $99^{[c]}$ \\
\hline 7 & B & 2.5 & 1.0 & 70 & $10^{[c]}$ \\
\hline 8 & B & 5.0 & 1.0 & 70 & $15^{[c]}$ \\
\hline 9 & C & 2.5 & 1.0 & 50 & $95(89)^{[d]}$ \\
\hline 10 & C & 5.0 & 1.0 & 50 & 99 \\
\hline 11 & C & 2.5 & 0.5 & 50 & 81 \\
\hline 12 & C & 2.5 & 0.75 & 50 & 84 \\
\hline 13 & C & 2.5 & 2.0 & 50 & 99 \\
\hline 14 & C & 1.0 & 1.0 & 70 & $99^{[c]}$ \\
\hline 15 & C & 2.5 & 1.0 & 70 & $99(71)^{[c, d]}$ \\
\hline
\end{tabular}

[a] General conditions: $0.5 \mathrm{mmol}$ phenylcarbamate, catalyst A-C (0-5 mol\%), $18 \mathrm{~h}, \mathrm{CH}_{3} \mathrm{CN}$. ${ }^{[b]}$ Conversion determined by ${ }^{1} \mathrm{H}$ NMR $\left(\mathrm{CDCl}_{3}\right)$ based on phenol formation. ${ }^{[c]}$ White precipitate noted. ${ }^{[d]}$ Isolated yield of oxazolidinone product in brackets.

Once discovered the best conditions for the conversion of $\mathrm{CHO}$ into product 1 using the most effective Al catalyst (i.e., C), we then started to investigate the scope of this process (Scheme 2). In general, this Al-mediated synthesis allows for a wide range of (functional) groups to be present in the epoxide substrate including alkene ( 2 and 4 ), alkyl halide (5), arylbromide (9), epoxidic (8), alkyne (13) and ether (10 and 13-15) groups. Of further note are the syntheses of oxazolidinones $\mathbf{1 1}$ and $\mathbf{1 6}$ and compounds 1-3 that comprise of an (a)cyclic dialkylsubstituted pattern. The alternative formation of acyclic and cyclic 4,5- and 5,5'-disubstituted oxazolidinone regio-isomers from aziridenes $/ \mathrm{CO}_{2}{ }^{[25-}$ ${ }^{27]}$, epoxide/isocyanate ${ }^{[28,29]}$ or propargylic amine $/ \mathrm{CO}_{2}{ }^{[30]}$ substrate combinations typically require harsher reaction conditions, a higher loading of catalyst and/or the use of stoichiometric/expensive additives and more elaborative procedures. The method disclosed here towards oxazolidinones 1-16 is operationally simple and allows for high conversion under attractive reaction conditions ( $50^{\circ} \mathrm{C}, 2 \mathrm{~mol} \%$ of catalyst). The majority of the oxazolidinone compounds could be isolated in good yields (up to 91\%) and high regioselectivity for the 5-isomer was noted with some exceptions (cf., the synthesis of $\mathbf{9 , 1 2}$ and 16). Since these reactions occur with inversion of configuration at the carbon centre at the initial stage of the reaction (i.e., nucleophilic attack of the carbamate reagent onto the epoxide-Al complex), those reactions that involve cis-configured carbon centres in the oxirane unit give rise to oxazolidinones with a trans disposition ( $c f$. , syntheses of 1-3 and 11a). Thus these conversions proceed with formal inversion unlike the reactions between isocyanates and epoxides. ${ }^{[29]}$ 


$$
\text { (2 equiv) }
$$

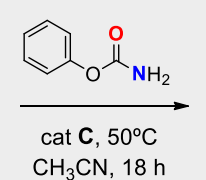

$\mathrm{O}$
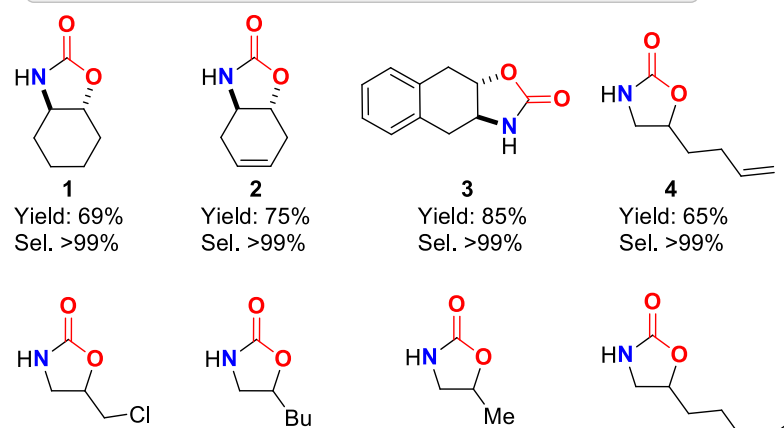

5

Yield: $82 \%$

Sel. $>99 \%$
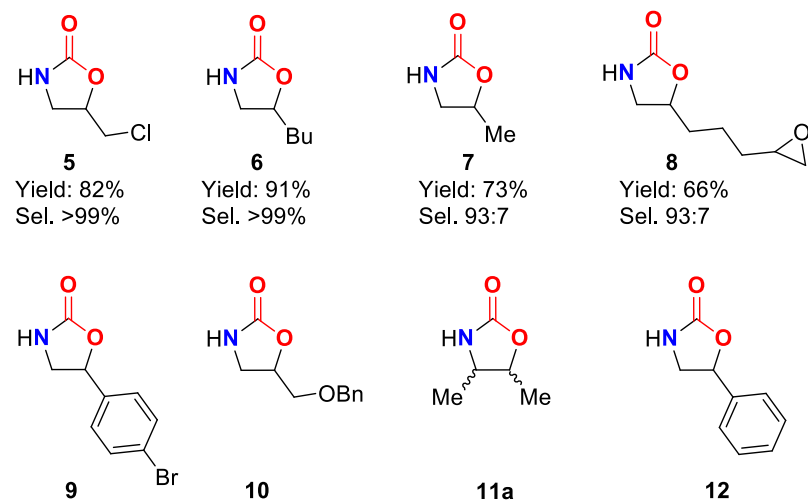

12

$70^{\circ} \mathrm{C}, 40 \mathrm{~h}$

Yield: $45 \%$

Sel. 82:18

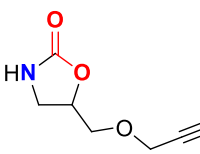

10
$70^{\circ} \mathrm{C}, 30 \mathrm{~h}$

Yield: $83 \%$

Sel. $>99 \%$

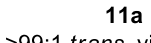

>99:1 trans, yield: $77 \%$$$
\text { 11b }
$$$$
\text { 11b }
$$

$70^{\circ} \mathrm{C}, 24 \mathrm{~h}$

Yield: $67 \%$

$>99: 1 \mathrm{cis}$, yield: $72 \%(66 \mathrm{~h}) \quad$ Sel. $67: 33$
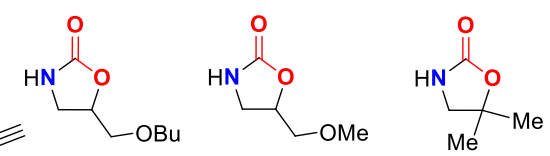

13

Yield: $76 \%$ Sel. 98:2

$$
\begin{aligned}
& 14 \\
& \text { Yield: } 75 \% \\
& \text { Sel. }>99 \%
\end{aligned}
$$

\section{5}

Yield: $89 \%$

Sel. $>99 \%$
Scheme 2. Synthesis of oxazolidinones 1-16 from various epoxides and phenylcarbamate catalysed by Al-aminotriphenolate complex C ( 2 mol\%). Reported here are isolated yields after column purification; selectivities refer to regio-isomers formed in the case of terminal epoxide conversion (note: only the major isomer is shown).

This inversion pathway was further substantiated by the synthesis of 11b (cis diastereoisomer of 11a; $d r>$ 99\%) that was prepared from the trans-epoxide.

The envisioned mechanism involves a nucleophilic attack of the phenyl carbamate on the coordinated epoxide producing a reactive alkoxide which is stabilized by the Al complex (Scheme 3 ) displaying dual character: the aminotriphenolate ligand acts here as a proton relay mediator increasing the nucleophilic character of the alkoxide which allows a more efficient ring-closing to occur releasing the final (configurationally inverted) product and a phenoxide as a leaving group. ${ }^{[31]}$ The formation of phenol, as a result of the phenoxide abstracting a proton, is easily recognized in the reaction mixture and thus allows for following the course of the process (see Table 1, Scheme 3).

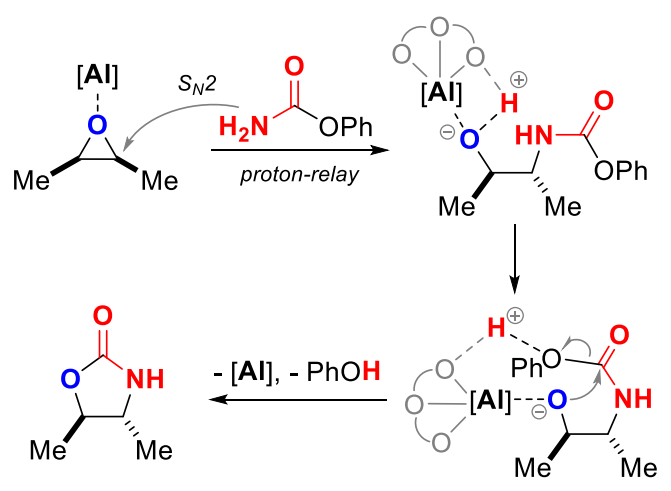

Scheme 3. Proposed mechanism leading to formal inversion in cis-2,3-dimethyloxirane.

The oxazolidinones 1-16 serve as useful precursors towards the formation of highly functional, nonsymmetrical ureas as shown in Scheme 4. Treatment of the respective oxazolidinone precursor with a suitable amine (1.2-4 equiv) allows for isolation of the urea product in high yield and chemo-selectivity. The aminolysis of the oxazolidinone occurs with exclusive preference for scission of the $\mathrm{NH}-\mathrm{C}=\mathrm{O}$ bond. The formation of ureas 17-24, 27 and 28 from bicyclic oxazolidinones proceeds generally under mild reaction conditions in the presence of a small excess of amine reagent in $\mathrm{CH}_{3} \mathrm{CN}$ solvent. This procedure is attractive, practical and proceeds with full conversion of the starting materials. In the case where oxazolidinones derived from terminal epoxides are used as starting materials (i.e., 25, 26 and 29-32), the aminolysis reaction required generally higher temperature, longer reaction times and a larger excess of amine reagent (4 equiv) under solvent-free conditions. In the latter cases, somewhat lower isolated yields are noted (up to $73 \%$ ).

The scope demonstrated in Scheme 3 illustrates that highly functionalized ureas can be prepared including those incorporating pyrrolidine (17), alkene $(19,23,25$ and 29), alkyne (20 and 31), heterocyclic 
$(17,24,25,27,31$ and 32), alkyl amine (28) and vicinal diol (26) groups. Note that urea $\mathbf{2 6}$ is derived from the oxazolidinone $\mathbf{5}$ which is based on the initial use of epichlorohydrin. The longer reaction time needed to convert $\mathbf{5}$ into $\mathbf{2 6}$ resulted in effective hydrolysis of the alkyl chloride fragment which is in line with the recorded NMR data and mass spectrometric analysis (see Supporting Information).

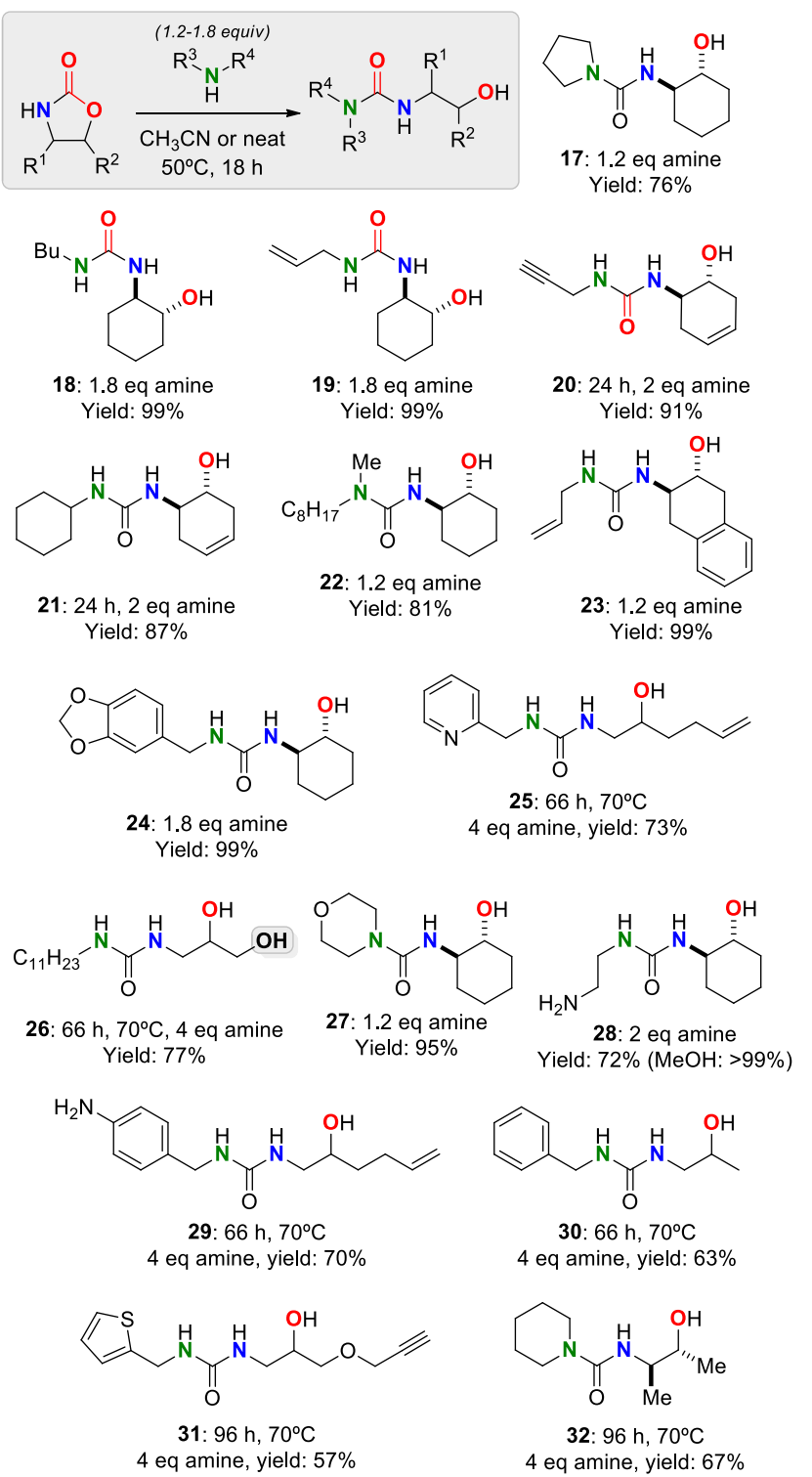

Scheme 4. Synthesis of nonsymmetrical ureas 17-32 from oxazolidinone precurcors and primary/secondary amines. Reported yields are isolated ones after column purification/crystallization.
Both primary as well as secondary amines (cf., syntheses of 17, 22, 27 and 32) react smoothly with the oxazolidinone precursors further amplifying the potential scope of this urea formation reaction. The molecular structure of urea $\mathbf{2 7}$ (Figure 2) was determined by X-ray diffraction. Compound $\mathbf{2 7}$ was derived from oxazolidinone 1 . Thus, the relative configuration of the urea and the free alcohol groups (trans) is further testament of the proposed inversion mechanism as depicted in Scheme 3.

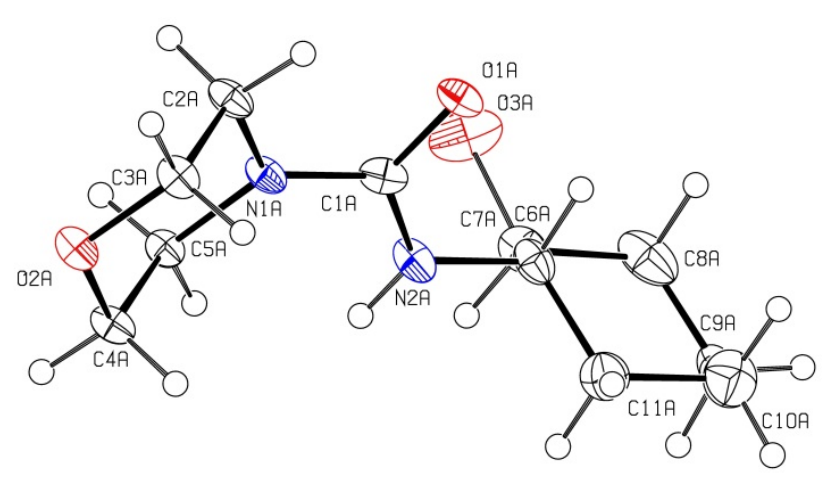

Figure 2. X-ray molecular structure determined for urea 27. Selected interatomic distances $(\AA \AA)$ and angles () with esd's in parentheses: $N(1 A)-C(1 A)=$ 1.366(5), $N(2 A)-C(1 A)=1.356(5), C(1 A)-O(1 A)=$ 1.248(5), N(2A)-C(6A) = 1.449(5); N(1A)-C(1A)-N(2A) $=117.8(4), N(1 \mathrm{~A})-\mathrm{C}(1 \mathrm{~A})-\mathrm{O}(1 \mathrm{~A})=120.4(4), \mathrm{N}(2 \mathrm{~A})-$ $\mathrm{C}(1 \mathrm{~A})-\mathrm{O}(1 \mathrm{~A})=121.8(4)$.

The diversity of functional groups in the presented scope of urea products has potential in postsynthetic modifications as shown in Scheme 5. To demonstrate further use of these scaffolds, urea $\mathbf{1 3}$ was subjected to "click" chemistry conditions using benzyl azide as coupling partner: this afforded the triazole 34 smoothly in $91 \%$ yield. ${ }^{[32]}$ The use of click reactions to functionalize polymer supports or other macromolecular structures is interesting for catalytic $^{[33]}$ and supramolecular applications ${ }^{[34,35]}$ and therefore this chemistry combined with suitable urea synthons opens up new opportunities in aforementioned areas. 


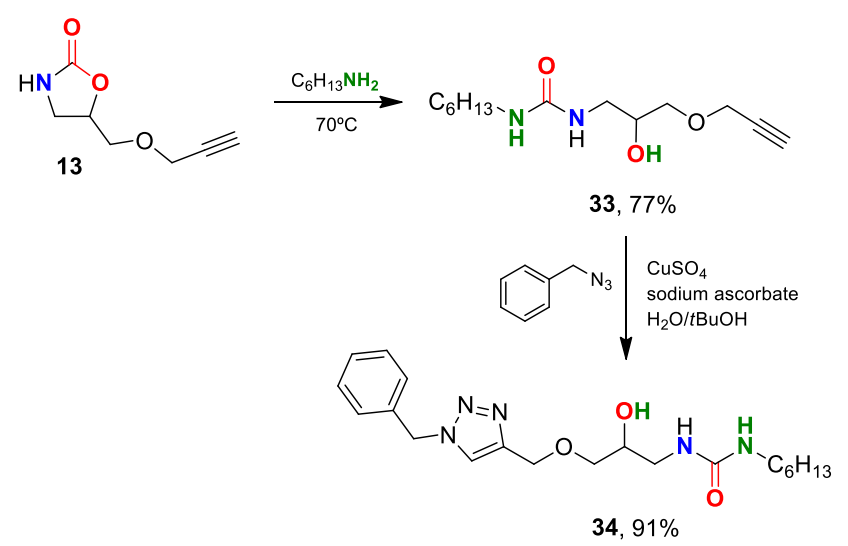

Scheme 5. Synthetic potential of urea 13.

\section{Conclusion}

We here disclose a simple but effective two-step method for the formation of highly functional, nonsymmetrical ureas from easy to prepare oxazolidinones. The cyclic carbamates are derived from cheap and readily available epoxides and phenylcarbamate mediated through Al-catalysis. The method is further characterized by its operational simplicity and wide scope in ureas that can be attained. Further to this, these functional ureas may serve as suitable scaffolds in organic synthesis which can be post-modified using "click" type reactions as demonstrated herein.

\section{Experimental Section}

\section{Oxazolidinone Synthesis}

Typically, phenyl carbamate $(1 \mathrm{mmol})$, the epoxide ( 2 $\mathrm{mmol}$ ), the aluminium catalyst ( $2 \mathrm{~mol} \%$ ) and acetonitrile $(2 \mathrm{~mL})$ were introduced into a glass vial ( $5 \mathrm{ml}$ ) equipped with a stirring bar. The vial was closed and introduced into a silicon oil bath preheated at the desired reaction temperature $(50-70 \circ \mathrm{C})$ and stirred for $18 \mathrm{~h}$. Once the reaction mixture had cooled down to r.t. the product was purified by flash-column chromatography following analysis. Full characterization data and copies of relevant spectra are provided in the Supporting Information.

\section{Urea Synthesis}

Typically, the oxazolidinone $(0.5 \mathrm{mmol})$ was introduced into an HPLC vial equipped with a stirring bar. For the oxazolidinones based on bicyclic scaffolds, the amine reagents were introduced (usually 1.2 equiv but was risen to 1.8 equiv when dealing with volatile amines) with addition of acetonitrile $(0.2 \mathrm{~mL})$ and heated to the desired temperature $\left(50^{\circ} \mathrm{C}\right)$ for $18 \mathrm{~h}$. Once the reaction mixture had cooled down to r.t. the pure product was isolated by evaporation of the excess of amine, and the crude ureas recrystallized from dichloromethane (ureas 20, 21, 23, 24, 27 and 28) or purified by flash column chromatography. The oxazolidinones with monocyclic scaffolds were treated with the respective amine reagent (4 equiv) under neat conditions and heated to $70 \circ \mathrm{C}$ for $66 \mathrm{~h}$. The products were then isolated by recrystallization from dichloromethane (for urea 29) or purified by flash column chromatography. Full characterization data and copies of relevant spectra are provided in the Supporting Information.

\section{Crystallographic Studies}

The measured crystal was stable under atmospheric conditions; nevertheless it was treated under inert conditions immersed in perfluoro-polyether as protecting oil for manipulation. Data Collection: measurements were made on a Bruker-Nonius diffractometer equipped with an APPEX II 4K CCD area detector, a FR591 rotating anode with Mo Ka radiation, Montel mirrors and a Kryoflex low temperature device $\left(T=-173^{\circ} \mathrm{C}\right)$. Full-sphere data collection was used with $\omega$ and $\phi$ scans. Programs used: Data collection Apex2 V2011.3 (Bruker-Nonius 2008), data reduction Saint+Version 7.60A (Bruker AXS 2008) and absorption correction SADABS V. 2008-1 (2008). Structure Solution: SHELXTL Version 6.10 (Sheldrick, 2000) was used. ${ }^{[36]}$ Structure Refinement: SHELXTL-97-UNIX VERSION. Crystal data for 27: $\mathrm{C}_{11} \mathrm{H}_{20} \mathrm{~N}_{2} \mathrm{O}_{3}, M_{\mathrm{r}}=228.29$, triclinic, $P-1, a=$ 6.4520(13) $\AA, b=9.1250(18) \AA, c=20.031$ (4) $\AA, a=$ 92.00 $, b=92.90 \circ, g=90.06 \circ, V=1177.1(4) \AA^{3}, Z=$ $4, \rho=1.288 \mathrm{mg} \cdot \mathrm{M}^{-3}, m=0.094 \mathrm{~mm}^{-1}, I=0.71073 \AA, T$ $=100(2) \mathrm{K}, F(000)=496$, crystal size $=0.15 \times 0.04 \times$ $0.01 \mathrm{~mm}, \theta(\min )=1.018^{\circ}, \theta(\max )=27.67^{\circ}, 5421$ reflections collected, 5421 reflections unique, $\mathrm{GoF}=$ $1.061, R_{1}=0.0812$ and $w R_{2}=0.1951[I>2 \sigma(I)], R_{1}=$ 0.1438 and $w R_{2}=0.2367$ (all indices), $\mathrm{min} / \mathrm{max}$ 
residual density $=-0.716 / 0.718\left[\mathrm{e} \cdot \AA^{-3}\right]$.

Completeness to $\vartheta\left(27.67^{\circ}\right)=98.9 \%$. CCDC number 1405849.

\section{Acknowledgements}

We thank ICIQ, ICREA and the Spanish Ministerio de Economía y Competitividad (MINECO) through Severo Ochoa Excellence Accreditation 2014-2018 (SEV-2013-0319) and project CTQ2014-60419-R for support. W.G. thanks the CELLEX foundation for financial support and V.L. wishes to acknowledge the Generalitat de Catalunya for an FI fellowship. Eduardo C. Escudero-Adán and Dr. Eddy Martin are thanked for the X-ray crystallographic analysis.

\section{References}

[1] N. Volz, J. Clayden, Angew. Chem. Int. Ed. 2011, 50, 12148-12155.

[2] S. J. Connon, Chem. Commun. 2008, 24992510.

[3] Y. Takemoto, Org. Biomol. Chem. 2005, 3, 4299-4306.

[4] S. Dawn, M. B. Dewal, D. Sobransingh, M. C. Paderes, A. C. Wibowo, M. D. Smith, J. A. Krause, P. J. Pellechia, L. S. Shimizu, J. Am. Chem. Soc. 2011, 133, 7025-7032.

[5] J. T. Su, P. Vachal, E. N. Jacobsen, Adv. Synth. Catal. 2001, 343, 197-200.

[6] For an example see: J. J. van Gorp, J. A. J. Vekemans, E.W. Meijer, J. Am. Chem. Soc. 2002, 124, 14759-14769.

[7] R. P. Sijbesma, F. H. Beijer, L. Brunsveld, B. J. B. Folmer, J. H. K. K. Hirschberg, R. F. M. Lange, J. K. L. Lowe, E. W. Meijer, Science 1997, 278, 1601-1604.

[8] One example: J. M. Rodriguez, A. D. Hamilton, Angew. Chem. Int. Ed. 2007, 46, 86148617.

[9] S. Hussain, P. R. Brotherhood, L.W. Judd, A. P. Davis, J. Am. Chem. Soc. 2011, 133, 1614-1617.
[10] K. J. Padiya, S. Gavade, B. Kardile, M. Tiwari, S. Bajare, M. Mane, V. Gaware, S. Varghese, D. Harel, S. Kurhade, Org. Lett. 2012, 14, 2814-2817.

[11] E. V. Vinogradova, B. P. Fors, S. L. Buchwald, J. Am. Chem. Soc. 2012, 134, 11132-11135.

[12] H. V. Le, B. Ganem, Org. Lett. 2011, 13, 25842585.

[13] H. Lebel, O. Leogane, Org. Lett. 2006, 8, 57175720.

[14] J. A. Fritz, J. S. Nakhla, J. P. Wolfe, Org. Lett. 2006, 8, 2531-2534.

[15] M. B. Bertrand, J. P. Wolfe, Tetrahedron 2005, 61, 6447-6459.

[16] For use of this aminolysis reaction in the preparation of urea-tethered neoglycoconjugates: $\mathrm{Y}$. Ichikawa, Y. Matsukawa, M. Isobe, J. Am. Chem. Soc. 2006, 128, 3934-3938.

[17] T. Yoshimitsu, T. Ino, T. Tanaka, Org. Lett. 2008, 10, 5457-5460.

[18] E. Bon, R. Réau, G. Bertrand, Tetrahedron Lett. 1996, 37, 1217-1220.

[19] G. Chen, P. Pan, Y. Yao, Y. Chen, X. Meng, Z. Li, Tetrahedron 2008, 64, 9078-9087.

[20] I. Maya, O. López, J. G. Fernández-Bolaños, I. Robina, J. Fuentes, Tetrahedron Lett. 2001, 42, 54135416.

[21] J. A. Birrell, E. N. Jacobsen, Org. Lett. 2013, 15, 2895-2897.

[22] C. J. Whiteoak, E. Martin, E. C. EscuderoAdán A. W. Kleij, Adv. Synth. Catal. 2013, 355, 22332239.

[23] C. J. Whiteoak, N. Kielland, V. Laserna, E. C. Escudero-Adán, E. Martin, A. W. Kleij, J. Am. Chem. Soc. 2013, 135, 1228-1231.

[24] V. Laserna, G. Fiorani, C. J. Whiteoak, E. Martin, E. Escudero-Adán, A. W. Kleij, Angew. Chem. Int. Ed. 2014, 53, 10416-10419. 
[25] M. T. Hancock, A. R. Pinhas, Tetrahedron Lett. 2003, 44, 5457-5460.

[26] J. Seayad, A. M. Seayad, J. K. P. Ng, C. L. L. Chai, ChemCatChem 2012, 4, 774-777.

[27] F. Fontana, C. C. Chen, V. K. Aggarwal, Org. Lett. 2011, 13, 3454-3457.

[28] P. Wang, J. Qin, D. Yuan, Y. Wang, Y. Yao, ChemCatChem 2015, 7, 1145-1151.

[29] T. Baronsky, C. Beattie, R. W. Harrington, R. Irfan, M. North, J. G. Osende, C. Young, ACS Catal. 2013, 3, 790-797.

[30] M. Shi, Y.-M. Shen, J. Org. Chem. 2002, 67, 16-21.

[31] We have monitored the reaction between cyclohexene oxide and phenyl carbamate by ${ }^{13} \mathrm{C}$ NMR spectroscopy and resonances of the product, the released phenol, phenyl carbamate and an additional species were noted. The latter may be assigned to an open carbamate structure prior to ring-closure leading to the cyclic carbamate, see the Supporting Information for details.
[32] F. Himo, T. Lovell, R. Hilgraf, V. V. Rostovtsev, L. Noodleman, K. B. Sharpless, V. V. Fokin, J. Am. Chem. Soc. 2005, 127, 210.

[33] C. J. Whiteoak, A. H. Henseler, C. Ayats, A. W. Kleij, M. A. Pericàs, Green Chem. 2014, 16, 1552.

[34] S. Dawn, M. B. Dewal, D. Sobransingh, M. C. Paderes, A. C. Wibowo, M. D. Smith, J. A. Krause, P. J. Pellechia, L. S. Shimizu, J. Am. Chem. Soc. 2011, 133, 7025.

[35] J. M. Roberts, B. M. Fini, A. A. Sarjeant, O. K. Farha, J. T. Hupp, K. A. Scheidt, J. Am. Chem. Soc. 2012, 134, 3334.

[36] G. M. Sheldrick, SHELXTL Crystallographic System, version 6.10; Bruker AXS, Inc.: Madison, WI, 2000. 


\section{FULL PAPER}

Expedient Synthesis of Functionalized Non-

Symmetrical Ureas from Oxazolidinone Precursors

Adv. Synth. Catal. 2015, 357, Page - Page

phenyl carbamate

Victor Laserna, Wusheng Guo, Arjan W. Kleij* 